hep-ph/0512332

\title{
TOWARDS A GEOMETRIC APPROACH TO THE FORMULATION OF THE STANDARD MODEL
}

\author{
V.G. Kadyshevsky*, M.D. Mateev* ${ }^{* \dagger}$, V.N. Rodionov ${ }^{\ddagger}$, A.S. Sorin* \\ * Joint Institute for Nuclear Research, \\ 141980 Dubna (Moscow Region), Russia \\ kadyshev@jinr.ru sorin@theor.jinr.ru \\ $\dagger$ University of Sofia St. Kliment Ohridsky, Sofia, Bulgaria \\ matey.mateev@gmail.com \\ $\ddagger$ Moscow State Geological Prospecting University, 118617 Moscow, Russia \\ vnrodionov@mtu-net.ru
}

\begin{abstract}
A geometric interpretation of the spontaneous symmetry breaking effect, which plays a key role in the Standard Model, is developed. The advocated approach is related to the effective use of the momentum 4spaces of the constant curvature, de Sitter and anti de Sitter, in the apparatus of quantum field theory.
\end{abstract}

\section{Introduction}

We sent this work for publication at the end of 2005 marked as the World Year of Physics. As its epigraph we could use the well-known citation of A. Einstein:

$$
\text { EXPERIMENT }=\text { GEOMETRY }+ \text { PHYSICS }
$$


This thesis has been convincingly confirmed in the special theory of relativity, the general theory of relativity and quantum theory. The universal constants $c, G$ and $\hbar$ playing a key role in these theories, allow simple interpretation in either a geometry context or group theoretical terms directly connected with geometry.

In the special theory of relativity the velocity of light $c$ appears in the definition of the metric of the pseudo-Euclidean space-time:

$$
d s^{2}=c^{2} d t^{2}-\left(d x_{1}\right)^{2}-\left(d x_{2}\right)^{2}-\left(d x_{3}\right)^{2} .
$$

The corresponding 3-dimensional velocity space has Lobachevsky geometry with negative curvature $-1 / c^{2}$.

In the general theory of relativity the space time carries Riemann geometry and the components of the Riemann metric tensor depending on the universal constants $c$ and $G$ as parameters play the role of gravitational potentials.

In quantum mechanics such observables as momentum, energy, and angular momentum are generators of the Galilean group. The Planck constant $\hbar$ is involved in them explicitly and determines the value of the quantum of angular momentum.

Taking into account the above considerations we may formulate the heuristic conclusion: the appearance of universal constants in the description of certain physical phenomena is a signal that in such cases an important role

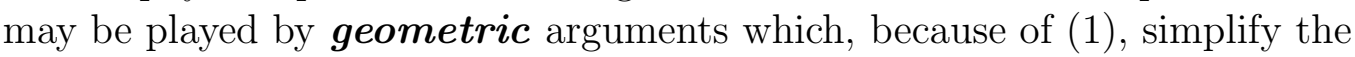
understanding of the physical essence of the considered processes.

To this end let us turn to the contemporary theory of elementary particles known as the Standard Model (SM). The notion "elementary particle" supposes that in accordance with present experimental data these objects do not have a composite structure and are described by local fields. The SM Lagrangian depends on a finite number of fields of this kind:

- three families of quark and lepton fields;

- four vector boson fields $W^{ \pm}, Z^{0}, \gamma$;

- an octet of gluon fields $g$;

- the hypothetic field of the Higgs boson $H$.

The $H$-boson has a different from zero vacuum expectation value

$$
\langle 0|H(x)| 0\rangle=h_{0}
$$

with dimension of mass. Thanks to this circumstance fields which have to be massive obtain masses proportional to $h_{0}$ when interacting with $H(x)$. 
As far as in the SM there are a finite number of particles, their masses are bounded from above.

The main idea underlying this work is a more general statement: mass spectrum of all particles described by local fields should cuts off on a certain mass $M$ :

$$
m \leq M \text {. }
$$

This universal parameter is called the fundamental mass. In other words, in fact we introduce $\boldsymbol{a}$ new notion of a local field, intrinsically consistent with the condition (4). Now objects for which $m>M$ cannot be considered as elementary particles, as to them does not correspond a local field.

The above-presented approach allows a simple geometric realization if one considers that the fundamental mass $M$ is the curvature radius of the momentum anti de Sitter 4 -space $(\hbar=c=1)$

$$
p_{0}^{2}-p_{1}^{2}-p_{2}^{2}-p_{3}^{2}+p_{5}^{2}=M^{2} \text {. }
$$

For a free particle, for which $p_{0}^{2}-\vec{p}^{2}=m^{2}$, the condition (4) is automatically satisfied on the surface (5). In the approximation

$$
\left|p_{0}\right|,|\vec{p}| \ll M, p_{5} \cong M
$$

the anti de Sitter geometry does not differ from the Minkowski geometry in four dimensional pseudo-Euclidean $p$-space.

However, it is much less obvious that in the momentum 4-space (5) one may fully develop the apparatus of quantum field theory, which after transition to configuration representation (with the help of a specific 5-dimensional Fourier transform) looks like a local field theoretical formalism in the four dimensional $x$-space [1]-[5]. It is fundamentally important that the new theory may be formulated in a gauge invariant way [1, 2, 4]). In other words, in the considered geometric approach there are conditions to construct an adequate generalization of the Standard Model, which gives one more illustration of the profundity of the Einstein idea (11). The following sections of this paper are devoted to this task.

At the end of this introduction we would like to note one important geometric point. The fact is that simultaneously with (5), in 5-dimensional $p$-space a second surface exists,

$$
p_{0}^{2}-p_{1}^{2}-p_{2}^{2}-p_{3}^{2}-p_{5}^{2}=-\mathfrak{M}^{2}
$$


on which the 4-dimensional de Sitter momentum space is realized. Although in this case the boundary on the particles mass (41) does not appear, in the flat limit (6) the de Sitter geometry, similarly to anti de Sitter one, coincides with the pseudo-Euclidean geometry of Minkowski $p$-space. Moreover, on the basis of (77) it is not difficult to develop the formalism of local gauge-invariant quantum field theory in configurational 4-space [1]- $[$ ] In what follows we shall see that this version of the theory will find its application in the realization of the Higgs mechanism in the developed approach.

\section{Spontaneous symmetry breaking in the case of neutral self-interacting scalar field}

Let us demonstrate that in the theory of self-interacting neutral scalar field $\varphi(x)$ developed on the basis of the de Sitter geometry (7) there is a spontaneous symmetry breaking of discrete symmetry (an analogue of $\varphi(x) \rightarrow$ $-\varphi(x))$. Moreover, after the transition to the new stable vacuum the Lagrangian of the system takes the form corresponding to the anti de Sitter case (5) and the fundamental mass $M$ is determined in terms of $\mathfrak{M}$. In other words, there is a phase transition from the de Sitter geometry to anti de Sitter geometry.

In accordance to 3], the free field Lagrangian for the massless neutral scalar field in the case (17) has the form:

$$
\mathcal{L}_{0}(x)=\frac{1}{2}\left(\frac{\partial \varphi(x)}{\partial x_{\mu}}\right)^{2}+\frac{\mathfrak{M}^{2}}{2}(\chi(x)-\varphi(x))^{2} \equiv \frac{1}{2}\left(\frac{\partial \varphi(x)}{\partial x_{\mu}}\right)^{2}-U_{0}(\varphi, x),
$$

where $\chi(x)$ is a neutral auxiliary field.

If we now introduce in (8) the simplest interaction in the form ${ }^{1}$ :

$$
\mathcal{L}_{\text {int }}(x)=-\frac{\lambda^{2}}{4}\left(\varphi^{2}(x)+\chi^{2}(x)\right)^{2},
$$

we obtain the following expression for the total density of the potential energy:

$$
U(x)=-\frac{\mathfrak{M}^{2}}{2}(\chi(x)-\varphi(x))^{2}+\frac{\lambda^{2}}{4}\left(\varphi^{2}(x)+\chi^{2}(x)\right)^{2} .
$$

\footnotetext{
${ }^{1}$ Let us note $[3]$ that in the limit $\mathfrak{M} \rightarrow \infty$, i.e. after the transition to the theory in the Minkowski $p$-space, the field variables $\varphi(x)$ and $\chi(x)$ coincide: $\varphi(x)=\chi(x)$.
} 
It is evident that the considered system has a nonstable vacuum state. The spontaneous transition to the new stable vacuum is accompanied by breaking of the discrete symmetry:

$$
\begin{gathered}
\varphi(x) \rightarrow-\varphi(x) \\
\chi(x) \rightarrow-\chi(x) .
\end{gathered}
$$

The standard procedure of substitution of $\varphi(x)$ and $\chi(x)$ by variables with zero vacuum expectation values

$$
\varphi^{\prime}(x)=\varphi(x)-\varphi_{0}=\varphi(x)-\frac{\mathfrak{M}}{\lambda}, \quad \chi^{\prime}(x)=\chi(x)-\chi_{0}=\chi(x)+\frac{\mathfrak{M}}{\lambda}
$$

leads to the total Lagragian of the system of the form:

$$
\begin{gathered}
\mathcal{L}(x)=\frac{1}{2}\left(\frac{\partial \varphi^{\prime}(x)}{\partial x_{\mu}}\right)^{2}-\frac{\mathfrak{M}^{2}}{2}\left(\chi^{\prime}(x)-\varphi^{\prime}(x)+2 \frac{\mathfrak{M}}{\lambda}\right)^{2}+ \\
+\frac{\lambda^{2}}{4}\left(\left(\varphi^{\prime}(x)-2 \frac{\mathfrak{M}}{\lambda}\right)^{2}+\left(\chi^{\prime}(x)+2 \frac{\mathfrak{M}}{\lambda}\right)^{2}\right)^{2}= \\
=\frac{1}{2}\left(\frac{\partial \varphi^{\prime}(x)}{\partial x_{\mu}}\right)^{2}-\frac{3}{2} \mathfrak{M}^{2}\left(\varphi^{\prime 2}(x)+\chi^{\prime 2}(x)\right)+\mathfrak{M}^{2} \varphi^{\prime}(x) \chi^{\prime}(x)+\mathcal{L}_{i n t}= \\
=\mathcal{L}_{0}\left(\varphi^{\prime}, \chi^{\prime}\right)+\mathcal{L}_{\text {int }}\left(\varphi^{\prime}, \chi^{\prime}\right) .
\end{gathered}
$$

On the other hand, in the theory of the neutral scalar field with mass $m$, based on the anti de Sitter geometry (5), the free field Lagrangian $\mathcal{L}_{0}(\varphi, \chi)$ has the form [3, 5]:

$$
\begin{gathered}
\mathcal{L}(x)=\frac{1}{2}\left(\frac{\partial \varphi(x)}{\partial x_{\mu}}\right)^{2}-\frac{m^{2}}{2} \varphi(x)^{2}-\frac{M^{2}}{2}(\chi(x)-\cos \mu \varphi(x))^{2}= \\
=\frac{1}{2}\left(\frac{\partial \varphi(x)}{\partial x_{\mu}}\right)^{2}-\frac{M^{2}}{2}\left(\varphi^{2}(x)+\chi^{2}(x)\right)+M^{2} \cos \mu \varphi(x) \chi(x), \\
\cos \mu=\sqrt{1-\frac{m^{2}}{M^{2}}} .
\end{gathered}
$$

Comparing $\mathcal{L}(x)$ (14) and $\mathcal{L}_{0}\left(\varphi^{\prime}, \chi^{\prime}\right)$ (13), we conclude that these two expressions are identical if one puts

$$
\mathfrak{M}^{2}=M^{2} \cos \mu ; \quad 3 \mathfrak{M}^{2}=M^{2} .
$$

Therefore, as a result of the spontaneous symmetry breaking the field $\varphi(x)$ obtains the mass

$$
m_{H}=\frac{2 \sqrt{2}}{3} M<M
$$


At the end of this paragraph we shall make two remarks:

1. In our approach all fields (including $\varphi(x)$ and, in a more general case, the Higgs field $H(x)$ ) before the symmetry breaking may be considered as massless. The point is that here the dimension of mass $[\mathrm{m}]$ is generated by the fundamental mass $\mathfrak{M}$.

2. In contrast to the standard approach we did not need to introduce a tachyon. In certain sense the role of a tachyon mass is played by the quantity $\mathfrak{M}$, which is the curvature radius of the de Sitter $p$-space.

\section{Spontaneous symmetry breaking in the case of interacting abelian vector and charged scalar fields}

Let us apply the developed in [1]-[5] methods to describe the interaction between a neutral abelian vector field and a charged scalar field with selfinteraction. As before we shall start with the de Sitter geometry (71). The total Lagrangian of the considered system in this case has the form ${ }^{2}$

$$
\begin{gathered}
\mathcal{L}(x)=\left[-\frac{1}{4} \overline{F_{K L}}\left(x, x_{5}\right) F^{K L}\left(x, x_{5}\right)+\right. \\
+2\left|\frac{\partial\left(e^{-i \mathfrak{M} x_{5}} A_{L}\left(x, x_{5}\right)\right)}{\partial x_{L}}-2 i \mathfrak{M} e^{-i \mathfrak{M} x_{5}} A_{5}\left(x, x_{5}\right)\right|^{2} \\
+\overline{D_{\mu} \varphi\left(x, x_{5}\right)} D^{\mu} \varphi\left(x, x_{5}\right)+\mathfrak{M}^{2}\left|\varphi\left(x, x_{5}\right)-\frac{i}{\mathfrak{M}} D_{5} \varphi\left(x, x_{5}\right)\right|^{2}- \\
\left.-\lambda^{2}\left(\left|\varphi\left(x, x_{5}\right)\right|^{2}+\frac{1}{\mathfrak{M}^{2}}\left|D_{5} \varphi\left(x, x_{5}\right)\right|^{2}\right)^{2}\right]_{x_{5}=0}, \quad K, L=0,1,2,3,5
\end{gathered}
$$

where we have introduced the covariant derivatives

$$
\begin{aligned}
D_{\mu} & =\partial_{\mu}+i q e^{-i \mathfrak{M} x_{5}} A_{\mu}\left(x, x_{5}\right), \\
D_{5} & =\partial_{5}+i q e^{-i \mathfrak{M} x_{5}} A_{5}\left(x, x_{5}\right) .
\end{aligned}
$$

Let us emphasize that in (17) the expression in square brackets is defined in five dimensional configuration space. The Lagrangian involves the variables $\frac{i}{\mathfrak{M}} \frac{\partial A_{\mu}}{\partial x_{5}}$ and $\frac{i}{\mathfrak{M}} \frac{\partial A_{5}}{\partial x_{5}}$, which have an auxiliary character. Moreover, (17) depends on the component $A_{5}$ which is a gauge degree of freedom. One may easily

\footnotetext{
${ }^{2}$ With the details one may be acquainted, for instance, in 4 .
} 
exclude all these quantities. As a result the Lagrangian takes the form

$$
\begin{aligned}
& \mathcal{L}(x)=-\frac{1}{4} F_{\mu \nu}(x) F^{\mu \nu}(x)+\overline{D_{\mu} \varphi(x)} D^{\mu} \varphi(x)+ \\
& +\mathfrak{M}^{2}|\varphi(x)-\chi(x)|^{2}-\lambda^{2}\left(|\varphi(x)|^{2}+|\chi(x)|^{2}\right)^{2}
\end{aligned}
$$

where

$$
\chi(x)=\left.\frac{i}{\mathfrak{M}} \frac{\partial \varphi\left(x, x_{5}\right)}{\partial x_{5}}\right|_{x_{5}=0} .
$$

Let us separate the real and imaginary parts in $\varphi$ and $\chi$,

$$
\varphi=\frac{1}{\sqrt{2}}\left(\varphi_{1}+i \varphi_{2}\right), \quad \chi=\frac{1}{\sqrt{2}}\left(\chi_{1}+i \chi_{2}\right),
$$

and pass to fields with zero vacuum expectation values, choosing the phases in a simple manner (compare with (12)).

$$
\begin{aligned}
\varphi_{1} & \rightarrow \varphi_{1}-\frac{\mathfrak{M}}{\lambda}, & \chi_{1} & \rightarrow \chi_{1}+\frac{\mathfrak{M}}{\lambda}, \\
\varphi_{2} & \rightarrow \varphi_{2}, & \chi_{2} & \rightarrow \chi_{2} .
\end{aligned}
$$

In the same way as in the usual theory one of the results of the spontaneous breaking of the gauge symmetry is a rearrangement of the field degrees of freedom with conservation of their number. The field $\varphi_{2}$ becomes the longitudinal component of the vector field $A_{\mu}$ and the latter obtains the mass

$$
m_{V}^{2}=\frac{\mathfrak{M}^{2} q^{2}}{\lambda^{2}} .
$$

Moreover, the quadratic in the scalar fields part of the Lagrangian has a structure corresponding to anti de Sitter geometry (77). Therefore, the parameter $\mathfrak{M}$ may be expressed, using (15), in terms of the fundamental mass $M$. As a result we obtain from (23) for the mass $m_{V}$ of the vector field

$$
\frac{m_{V}^{2}}{M^{2}}=\frac{q^{2}}{3 \lambda^{2}} \text {. }
$$

Since in the geometry (5) the mass of a particle cannot exceed the fundamental mass $M$, from (24) it follows the relation between the coupling constants $q$ and $\lambda$

$$
q \leq \sqrt{3} \lambda .
$$

In the next paper we shall consider the mechanism of spontaneous symmetry breaking of nonabelean gauge symmetry in the framework of the present approach. 
Acknowledgements The authors would like to express their sincere gratitude to I. Aref'eva and I. Volovich for fruitful discussions of different aspects of this work.

The study was partly supported by the Russian Foundation for Basic Research (project N05-02-16535) and the Council of the President of the Russian Federation for support of Young Russian Scientists and Leading Scientific Schools (project NNSh-2027.2003.2)

\section{References}

[1] Kadyshevsky V.G., Nucl. Phys. 1978, B141, p 477; in Proceedings of International Integrative Conference on Group theory and Mathematical Physics, Austin, Texas, 1978; Fermilab-Pub. 78/70-THY, Sept. 1978; Phys. Elem. Chast. Atom. Yadra, 1980, 11, p5.

[2] Kadyshevsky V.G., Mateev M. D., Phys. Lett., 1981, 106B, p139.

[3] Kadyshevsky V.G., Mateev M. D., Nuovo Cimento, 1985, A87, p324.

[4] Chizhov M. V., Donkov A.D.,Kadyshevsky V.G., Mateev M. D., Nuovo Cimento, 1985, A87, p350.

[5] Kadyshevsky V.G., Phys. Part. Nucl. 1998, 29, p 227. 